\title{
Vaccination of dogs and cats: still relatively controversial
}

\author{
Vaccination of dogs and cats: still relatively controversial: The open letter to the Vaccination Guidelines Group \\ (VGG) of the World Small Animal Veterinary Association (WSAVA)
}

S.T. Orlova, postgraduate student of the Department of Epizootiology and Organization of Veterinary Affairs, veterinary practitioner of the "Clinic of veterinary medicine in Zvenigorod»(https://orcid.org/0000-0002-0830-1364, werta.sto@ mail.ru), A.A. Sidorchuk', doctor of veterinary Sciences, professor, Department of Epizootiology and Organization of Veterinary Affairs (saa48@mail.ru).

${ }^{1}$ Moscow State Academy of Veterinary Medicine and Biotechnology named after K.I. Skryabin (23a, Ac. Skryabin str., Moscow, 109472). ${ }^{2}$ Clinic of veterinary medicine in Zvenigorod (8, Pochtovaya str., Zvenigorod, 143180, Russia).

Although there have been no significant changes or breakthroughs in the production of vaccines for dogs and cats in recent years, the international guidelines for their vaccination, including the WSAVA Vaccination Guidelines, are updated with an average frequency of every five years. We believe this is primarily due to the fact that the world veterinary community has not yet developed a common view on this problem. Looking forward to the next update of the WSAVA Guidelines for the vaccination of dogs and cats in 2020, we decided to appeal to the members of the Vaccination Guidelines Group (VGG) of the World Small Animal Veterinary Association (WSAVA) in the open press to share with them our doubts about the certain items of the latest version of the 2015 Guidelines, as well as ask them some questions in the hope that the next update will cover all of them.

Key words: dogs, cats, vaccination, global Guidelines for the vaccination of dogs and cats, vaccination schedule, «overvaccination», «low-risk» and «higher-risk» animals, «infectious» and «non-infectious» vaccines.

Vaccination of pet small animals has attracted keen interest and lively debates over the last couple of decades not only among breeders but also among veterinarians. We are very grateful to various international organisations, including the American Academy of Feline Practitioners (AAFP), the American Animal Hospital Association (AAHA), and the Advisory Board on Cat Diseases (ABCD), for attempting to standardise this procedure and clarify the vaccination field for practitioners. We would like to express special gratitude to the Vaccination Guidelines Group (VGG) of the World Small Animal Veterinary Association (WSAVA). The VGG developed the guidelines for the vaccination of dogs and cats for global application back in 2007, and the guidelines were subsequently updated and expanded in 2010 and 2015 [1...3]. We sincerely hope that once a 5 -year period is expired, the next updated version of the WSAVA Vaccination Guidelines will be released in 2020. We were involved in the official translations to Russian of the last two versions (2010 and 2015) posted on the WSAVA website. Of course, as translators of the specified documents, we read the text ourselves much more attentively and know it much better than ordinary readers do. Since the VGG does not include any representatives from Russia or other countries of the former Soviet Union, the authors of the guidelines can hardly take into account the nuances of the epidemic situation and the keeping of animals in this vast territory occupying approximately $1 / 6$ of the land. Therefore, we would like to express our opinion on some issues covered in the guidelines on behalf of our compatriots hoping that the VGG will consider our comments when developing the 2020 version.

We are extremely grateful to the members of the VGG for developing a new classification scheme for evidencebased veterinary medicine (EBVM) related to vaccinology. It is unfortunate that the current situation renders "category 1 evidence: a recommendation supported by the peer-reviewed scientific publication of either experimental or field data" as the first-class (highest) level of evidence in this area of veterinary medicine. In certain fields of human medicine, the latter corresponds approximately to the third (penultimate) category of evidence ( «non-randomised clinical trials on a limited number of patients"), whereas in contrast "the best of the large-scale, double-blind, placebo-controlled studies" represents the first category that resides at the top of the pyramid. Arguably, in this area, veterinary and human medicine are separated by a chasm. A hope remains that the joint efforts of researchers around the world will enable veterinary medicine to rise to the equally high level one day.

The main issue that provokes our objections is the new basic vaccination schedule for puppies and kittens during their first 6 months of life. It is suggested that "vaccination started in a 6- or 7-week old puppy or kitten, might now entail up to five vaccine visits in the first 6 months of life». The VGG declares that the main aim of administration of these vaccines "is to ensure that a protective immune response develops in any animal that may have failed to respond to any of the vaccines in the primary core series, rather than necessarily 'boosting' the immune response". For puppies and kittens entering a shelter, the VGG proposes administration of core vaccines up to 8 times in total during the first 6 months of their lives: "core vaccination may be started as early as $4 . . .6$ weeks of age, and (where funding permits) revaccination should be every 2 weeks until the animal reaches 20 weeks of age". It appears rather strange against the background of the assertion that "we should aim to reduce the 'vaccine load' on individual animals in order to minimise the potential for adverse reactions to vaccine products and reduce the time and financial burden on clients and veterinarians of unjustified veterinary medical procedures». By reducing the number of revaccinations of adult animals via increasing the interval between them from one year to three years, the VGG members suggest increasing the 'vaccination load' several-fold for an actively growing puppy or kitten during the first months of life. Isn't this exactly the very 
same 'over-vaccination' that the owners of small companion animals are so scared of?

In addition, a veterinarian who adheres to such recommendations will lose credibility in the eyes of his clients relative to colleagues who normally administer two doses of core vaccines to a puppy or a kitten $2 \ldots 4$ weeks apart and revaccinate the animal after 1 year or at the age of 1 year according to the manufacturers' data sheets. After all, the client sees that the animals of other owners around him that were vaccinated only twice during the first year of their lives feel great. Of course, there is a certain proportion of animals that will not respond to vaccination administered in accordance with the standard two-shot schedule, but it is important to bear in mind that a certain proportion of them may be poor responders or non-responders by nature (their immune systems intrinsically fail to recognise the vaccine antigens). What is the real percentage of puppies and kittens vaccinated according to the old standard schedule that become sick between the traditional 3 months and $12 \ldots 15$ months? Why complicate the schedule so much if there is very few of them? Why torment the owners of all animals because of such small percentage? Furthermore, in low-income countries (e.g. Russia), informing the owner of the need to administer the vaccines to his or her puppy or kitten 5 times over 6 months may lead to complete refusal to vaccinate attributed to economic reasons. Perhaps it would be prudent to add a simple booster at 6 months to the standard manufacturers' scheme reducing the number of vaccine injections during the first year of life to just 3.

In our opinion, the rule of revaccination at the age of 6 months should be extended to vaccines against rabies as cases of the disease developing in animals between vaccination at the age of 3 months and revaccination after 1 year have been reported [4].

Different schedules of vaccination of "low-risk animals" and "higher-risk animals" represents another important issue. From our point of view, either the animal is protected or it is unprotected and everything else is irrelevant. What does the concept "a low-risk animal" mean? Does it mean that we have vaccinated the animal enough and expect it not get sick when exposed to infectious agents found in its habitat? If such an indoor animal is in fact unprotected when facing a real pathogen, why has its owner paid money for vaccination at all? Is this fair to the owner? Perhaps it is logical to develop REAL protection schemes using the shelter populations and then extend them to all other animals.

An excellent proposal was to divide all vaccines into "core vaccines" and "non-core vaccines". At the first glance, the introduction of such concepts as "infectious vaccines" and "non-infectious vaccines" in nature also seems attractive. However, more detailed reflection raises doubts. The term 'infectious vaccine' implies the ability of the vaccine pathogen "to induce immunity by inducing low-level infection and replicating within the animal, without producing significant tissue pathology or clinical signs of infectious disease». In practice, this only includes live vaccines (i.e. "modified live" or "attenuated" vaccines). The authors' explanation that recombinant vaccines should also be referred to as 'infectious' "because the protein antigens they encode or contain can be expressed by antigen presenting cells which will induce all forms of acquired immunity, much like the 'infectious vaccines" seems unconvincing to us. In a strict sense, live vector organisms of recombinant vaccines are not able "to induce immunity by inducing lowlevel infection and replicating within the animal, without producing significant tissue pathology or clinical signs of infectious disease" and, accordingly, recombinant vaccines do not fall under the definition of 'infectious vaccines'. It turns out that the concept of 'infectious vaccines' includes only live modified (attenuated) vaccines, and the concept of 'non-infectious vaccines' includes all other forms. We believe that introduction of an additional term exclusively for live vaccines makes no sense at all. Furthermore, these terms can scare less educated owners - it is suggested that the vaccine 'infects' their beloved pets!

It is wonderful that the Guidelines-2015 became a fullyfeatured vaccination review. Most of the recommendations are supported by references to pertinent scientific publications that the reader can easily review if necessary. We did not quite understand a few peripheral issues and request that references to scientific sources of such information be provided in the next editions of the guidelines, if possible.

Firstly, we are concerned about the duration of the period when absorption of colostrum antibodies via the intestine of a newborn puppy or kitten into systemic circulation is possible. In the 2010 version, 72 hours were specified, while 24 hours were specified in the 2015 version (Vaccination Guidelines 2015; Frequently Asked Questions, Question No 71, page E40). Is it the research data or the personal opinion of the experts?

And secondly, we were surprised by the message that "although it has been assumed that revaccination prior to pregnancy will boost the antibody level in the bitch so that she can transfer a higher level of the MDA to the pup, revaccination, especially with infectious/MLV vaccines, often provides no increase (boost) in her antibody because her existing antibody neutralises the vaccine at time of injection, so it does not infect or cause an immune response, which is what is required to provide immunity and to increase the antibody level" (Vaccination Guidelines for Owners and Breeders of Cats and Dogs 2015, page 45). Aren't the titres increased after revaccination in all animals and people with a normally functioning immune system?

In conclusion, we would like to sincerely thank once again the members of the WSAVA VGG for their regularly doing great work. We hope that our doubts and questions will be completely dispelled in the next update of the WSAVA Guidelines for the vaccination of dogs and cats for global application.

References

1. Day M.J., Horzinek M.C., Schultz R.D., Vaccination Guidelines Group (VGG) of the World Small Animal Veterinary Association (WSAVA), Guidelines for the vaccination of dogs and cats, Compiled by the Vaccination Guidelines Group (VGG) of the World Small Animal Veterinary Association (WSAVA). Journal of Small Animal Practice, 2007, No. 48, pp. 528-541.

2. Day M.J., Horzinek M.C., Schultz R.D., Vaccination Guidelines Group WSAVA guidelines for the vaccination of dogs and cats. Journal of Small Animal Practice, 2010, No. 51, pp. 1-32

3. Day M.J., Horzinek M.C., Schultz R.D., Squires R.A., Vaccination Guidelines Group (VGG) of the World Small Animal Veterinary Association (WSAVA), WSAVA Guidelines for the vaccination of dogs and cats, Journal of Small Animal Practice, 2016, No. 57, pp. 4-8 \& E1-E45.

4. Wilson P.J., Oertli E.H., Hunt P.R., Sidwa T.J., Evaluation of a postexposure rabies prophylaxis protocol for domestic animals in Texas: 2000-2009, Journal of the American Veterinary Medical Association, 2010, No. 237, pp. 1395-1401.

The Russian translation of the WSAVA Vaccination Guidelines (2015): https://www.wsava.org/WSAVA/media/Documents/ Guidelines/WSAVA-Vaccination-Guidelines-2015_RUS.pdf 